\title{
Targeting mutant BRAF in metastatic melanoma
}

Oncogenic mutations of BRAF, the most frequently mutated protein kinase in human cancers, are particularly common in melanoma and found in more than $40 \%$ of patients. The development of inhibitors targeting BRAF is of particular interest as the growth of $B R A F$ mutant melanoma cells seems to be dependent on the RAF-MEK-ERK signalling pathway. Now, reporting in Nature, Bollag and colleagues describe PLX4032, a potent inhibitor of mutant BRAF that has achieved a remarkable $81 \%$ response rate in Phase I trials of patients with $B R A F$-mutant metastatic melanoma.

PLX4032 is related to the previously reported compound PLX4720 and was developed using a crystallography-guided approach. This allowed the optimization of the compound for preferential binding to mutant BRAF (bearing the V600E mutation) over wild-type BRAF. The authors present the co-crystal structure of PLX4032 with the kinase

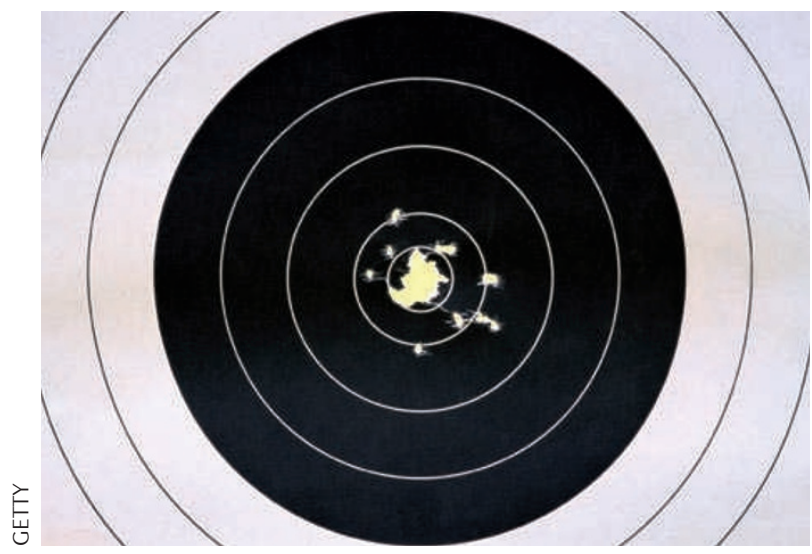

domain of BRAF (V600E) and demonstrate potent tumour growth inhibition in PLX4032-treated animals with $B R A F$-mutant colon cancer xenografts. PLX4032 was chosen for further development over PLX4720 because of its favourable pharmacokinetic properties in dogs and monkeys. Moreover, safety studies of PLX4032 in dogs and rats showed no signs of toxicity when doses up to $1,000 \mathrm{mg}$ per $\mathrm{kg}$ per day were tested.

On the basis of these data, Phase I clinical trials in patients with melanoma were initiated. As initial trials using oral capsules of a crystalline formulation of the drug only achieved low drug exposures and no clinical responses, the drug was reformulated into micro-precipitated bulk powder. This allowed for substantially improved bioavailability. A dose of $960 \mathrm{mg}$ twice daily was identified as the maximum tolerated dose, with limiting toxicities including fatigue, rash and joint pain. As observed with other RAF inhibitors, skin lesions were noted in a subset of patients, which were treated by resection.

Out of 32 patients treated at the maximum tolerated dose, 24 had partial responses and two had complete responses. Interestingly, two patients that responded particularly well were found to have the V600K mutation rather than the V600E mutation. Measurements of cytoplasmic and nuclear phospho-ERK in paired biopsy specimens from the patients - with one taken before and one taken 2 weeks into treatment
- showed that tumour regression correlated with greater than $80 \%$ inhibition of cytoplasmic ERK phosphorylation. Levels of nuclear phospho-ERK were more sensitive to drug inhibition but were less predictive of a clinical response.

These results indicate that clinical responses to BRAF inhibitors require a broad target inhibition and support the hypothesis that mutant BRAF is a dominant driver of tumour growth and maintenance. This is interesting as BRAF (V600E) mutations are likely to be initiating events, as a vast majority of benign nevi harbour the same mutation. The durability of the response is still under evaluation but the median progression-free survival is estimated to be at least 7 months, compared with 2 months in historical analyses. The mechanisms of resistance development are currently under investigation but with its promising safety and efficacy profile PLX4032 might be particularly suitable for combination therapy with other targeted agents, immunotherapy or chemotherapy.

\section{Alexandra Flemming}

ORIGINAL RESEARCH PAPER Bollag, G. et al. Clinical efficacy of a RAF inhibitor needs broad target blockade in BRAF-mutant melanoma. Nature 467, 596-599 (2010)

FURTHER READING Yang, H. et al. RG7204 (PLX4032), a selective BRAF ${ }^{\mathrm{V} 600 \mathrm{E}}$ inhibitor, displays potent antitumor activity in preclinical melanoma models. Cancer Res. 70, 5518-5527 (2010) | Flaherty, K. et al. Inhibition of mutated, activated BRAF in metastatic melanoma. N. Engl.J. Med. 363, 809-819 (2010) 